\title{
Steady-state solutions for a suspension bridge with intermediate supports
}

Claudio Giorgi* and Elena Vuk

${ }^{\text {*Correspondence: }}$ claudio.giorgi@ing.unibs.it DICATAM, Università degli Studi di Brescia, Via Valotti 9, Brescia, 25133, Italy

\begin{abstract}
This work is focused on a system of boundary value problems whose solutions represent the equilibria of a bridge suspended by continuously distributed cables and supported by $M$ intermediate piers. The road bed is modeled as the junction of $N=M+1$ extensible elastic beams which are clamped to each other and pinned at their ends to each pier. The suspending cables are modeled as one-sided springs with stiffness $k$. Stationary solutions of these doubly nonlinear problems are explicitly and analytically derived for arbitrary $k$ and a general axial load $p$ applied at the ends of the bridge. In particular, we scrutinize the occurrence of buckled solutions in connection with the length of each sub-span of the bridge.
\end{abstract}

MSC: Primary 35G30; secondary 74G05; 74G60; 74K10

Keywords: extensible elastic beam; suspension bridge; boundary value problems for nonlinear higher-order PDE; bifurcation and buckling

\section{Introduction}

In this paper, we investigate the solutions of a system of one-dimensional nonlinear problems describing the steady-states of an extensible elastic suspension bridge with $M$ intermediate supports (piers). In particular, we assume that the road bed of the bridge (deck) is composed of $N=M+1$ extensible elastic beams which are clamped to each other, pinned at their ends to each pier and suspended by a continuous distribution of flexible elastic cables. On account of the midplane stretching of the beams due to their elongations, a geometric nonlinearity appears into the bending equations of the deck.

In the case of a bridge with a single span (no intermediate pier), the problem can be recast into a non-dimensional setting, where its length is supposed to be unitary, for simplicity. Let $u=u(x):[0,1] \rightarrow \mathbb{R}$ be a non-dimensional field which accounts for the downward deflection of the bridge in the vertical plane with respect to its reference configuration, and let $u^{+}$stand for its positive part, namely

$$
u^{+}= \begin{cases}u & \text { if } u \geq 0 \\ 0 & \text { if } u<0 .\end{cases}
$$

Assuming that both ends of the bridge are pinned, the equation of the bending equilibrium looks like (see [1])

$$
\left\{\begin{array}{l}
u^{\prime \prime \prime \prime}+\left(p-\left\|u^{\prime}\right\|_{L^{2}(0,1)}^{2}\right) u^{\prime \prime}+k u^{+}=0 \\
u(0)=u(1)=u^{\prime \prime}(0)=u^{\prime \prime}(1)=0
\end{array}\right.
$$

\section{管 Springer}

(c) 2013 Giorgi and Vuk; licensee Springer. This is an Open Access article distributed under the terms of the Creative Commons Attribution License (http://creativecommons.org/licenses/by/2.0), which permits unrestricted use, distribution, and reproduction in any medium, provided the original work is properly cited. 


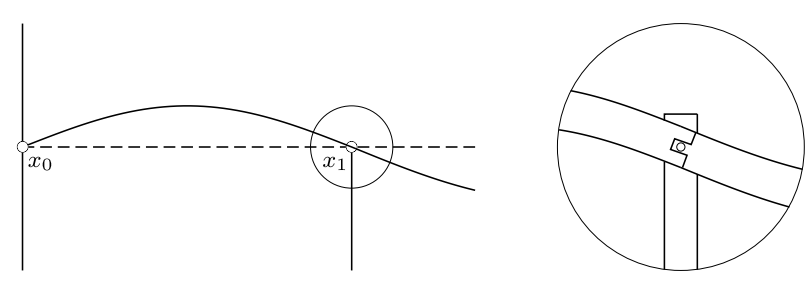

Figure 1 The joint connecting two consecutive sub-spans and the intermediate pier.

The term $-k u^{+}$accounts for the restoring force due to one-sided springs which models the supporting cables. Since we confine our attention to stationary conditions, we neglect the dynamical coupling between the deck and the main cable. The constant $p$ represents a non-dimensional measure of the axial force acting at the ends of the span in the reference configuration. Accordingly, $p$ is negative when the span is stretched, positive when compressed. The symbol ' represents the derivative with respect to the argument.

Assume now that the bridge span is composed of $N$ extensible beams whose internal ends are hinged to $M=N-1$ intermediate piers. Let $x_{i} \in(0,1), i=1, \ldots, M$, be the points at which these supports are located and let

$$
0=x_{0}<x_{1}<\cdots<x_{N-1}<x_{N}=1
$$

We denote by $I_{n}=\left(x_{n-1}, x_{n}\right), n=1, \ldots, N$, the corresponding intervals between two subsequent piers and by $\left|I_{n}\right|$ the corresponding length, so that

$$
\sum_{n=1}^{N}\left|I_{n}\right|=1
$$

According to the assumptions, the deflection $u$ of the whole span obeys

$$
u\left(x_{\ell}\right)=u^{\prime \prime}\left(x_{\ell}\right)=0, \quad \ell=0,1, \ldots, N .
$$

Furthermore, we assume that consecutive sub-spans are mutually clamped at the common end, which in turn implies the continuity of $u^{\prime}$ across the supported points $x_{i}, i=1, \ldots, M$ (see Figure 1).

Let $u_{n}=u_{n}(x): I_{n} \rightarrow \mathbb{R}, n=1, \ldots, N$, account for the downward deflection of the $n$th beam in the vertical plane, and let $p_{n}$ be the axial load acting on it. Of course, $u_{n}$ can be viewed as the restriction of $u$ on $I_{n}$, namely $u_{n}=\left.u\right|_{I_{n}}$. Then, taking advantage of the nonlinear model (1.1) for a single supported span, each $u_{n}$ solves the following boundary value problem:

$$
\left\{\begin{array}{l}
u_{n}^{\prime \prime \prime \prime}+\left(p_{n}-\left\|u_{n}^{\prime}\right\|_{L^{2}\left(I_{n}\right)}^{2}\right) u_{n}^{\prime \prime}+k u_{n}^{+}=0, \quad \text { in } I_{n} \\
u_{n}\left(x_{n-1}\right)=u_{n}\left(x_{n}\right)=u_{n}^{\prime \prime}\left(x_{n-1}\right)=u_{n}^{\prime \prime}\left(x_{n}\right)=0 .
\end{array}\right.
$$

Such solutions must fulfill the mutual groove condition

$$
u_{i}^{\prime}\left(x_{i}\right)=u_{i+1}^{\prime}\left(x_{i}\right), \quad i=1,2, \ldots, M .
$$


We finally observe that the total elongation of the span equals the sum of the elongations of each beam, namely

$$
\left\|u^{\prime}\right\|_{L^{2}(0,1)}^{2}=\sum_{n=1}^{N}\left\|u_{n}^{\prime}\right\|_{L^{2}\left(I_{n}\right)}^{2} .
$$

Throughout the paper, we assume a uniform distribution of the axial load, that is, $p_{n}=p$, $n=1,2, \ldots, N$. In addition, we let

$$
k=\kappa \pi^{4}, \quad \kappa \in \mathbb{R}^{+}, \quad p=b \pi^{2}, \quad b \in \mathbb{R} .
$$

Our aim is to scrutinize the existence of suitable buckled solutions for $u$, which can be obtained by joining buckled solutions for $u_{n}$ on each sub-span, $n=1,2, \ldots, N$. For later convenience, we denote such solutions by $u_{(N)}=\left(u_{1}, \ldots, u_{N}\right)$. We prove that they exist provided that the lengths of the sub-spans are properly chosen.

\subsection{Early contributions}

In recent years, an increasing attention has been payed to the analysis of buckling, vibrations and post-buckling dynamics of nonlinear beam models (see, for instance, [2, 3]). As far as we know, most of the papers in the literature deal with approximations and numerical simulations, and only few works are able to derive exact solutions (see [4-7]).

The investigation of solutions to BVP (1.1), in dependence on $p$, represents a classical nonlinear buckling problem in the literature on structural mechanics. The notion of buckling, introduced by Euler more than two centuries ago, describes a static instability of structures due to in-plane loading. In this respect, the main concern is to find the critical buckling loads, at which a bifurcation of solutions occurs, and their associated mode shapes, called postbuckling configurations. In the case $k=0$, a careful analysis of the corresponding buckled stationary states was performed in [7] for all values of $p$ in the presence of a source with a general shape (see also [8]). By replacing $u^{+}$with $u$ in (1.1), we obtain a simpler model which was scrutinized in $[1,5]$.

In addition, it is worth noting that (1.1) represents the static counterpart of quite many different evolution problems arising both from elastic, viscoelastic and thermo-elastic theories. An example is the following quasilinear equation:

$$
\partial_{t t} u+\partial_{x x x x} u+\left(p-\left\|\partial_{x} u\right\|_{L^{2}(0,1)}^{2}\right) \partial_{x x} u+\partial_{t} u+k u^{+}=f,
$$

which is obtained by matching the modeling of the extensible elastic beam (see $[9,10])$ with the well-known equation describing the motion of a damped suspension bridge (see $[11,12])$

$$
\partial_{t t} u+\partial_{x x x x} u+\partial_{t} u+k u^{+}=f .
$$

Free and forced vibrations of $(1.5)$ were recently scrutinized in $[13,14]$, whereas the longtime behavior of (1.4) was described in [15] for all values of $p$.

Obviously, solutions to BVP (1.1) represent the steady states of a lot of models more general than (1.4), for instance, when either the rotational inertia (as in the Kirchhoff theory) 
or some kind of damping are taken into account. In particular, (1.1) works either when external viscous forces are added or when some structural dissipation phenomena occur in the deck, as in thermoelastic and viscoelastic beams (see, for instance, [16-18]).

When the geometric nonlinear term into (1.1) is disregarded, the existence of nontrivial (positive) solutions to the corresponding system were established in [19] by the variational method. Therein, some nonlinearly perturbed versions were also scrutinized, but the set of assumptions made there no longer holds when the full model is considered.

\subsection{Outline of the paper}

To the best of our knowledge, this is the first paper in the literature dealing with exact solutions to the doubly nonlinear BVPs (1.2), even for $N=1$. As is well known, the analysis of the corresponding set $\mathcal{S}_{\kappa}$ of their stationary solutions takes a great importance in the longterm dynamics of the corresponding evolution system, especially when its structure is nontrivial [20]. The main results of this paper concern the steady states analysis of a bridge with $N=1,2,3$ sub-spans and are stated in Section 2. In Section 2.1 we scrutinize the case of a single span without piers $(N=1)$ and we prove that increasing the value of the lateral load $p$, first a negative $u_{(1)}^{-}$, then a positive $u_{(1)}^{+}$buckled solution appear at equilibrium. In Section 2.2 a bridge with a single pier $(N=2)$ is considered. When the position of the pier is allowed to be asymmetric $\left(\left|I_{1}\right| \neq\left|I_{2}\right|\right)$, we establish a condition on $\varepsilon=\left|I_{1}\right|$ in order that buckled static solutions exist. In particular, we prove that $\varepsilon \rightarrow 1 / 2$ as $k \rightarrow 0$. Taking advantage of these results, the analysis of a bridge with two symmetrically-placed piers $(N=3)$ is performed in Section 2.3, where buckled static solutions are proved to exist provided that $\left|I_{1}\right|=\left|I_{3}\right| \neq\left|I_{2}\right|$ fulfills a suitable condition. In Section 3 we deal with the general problem of a bridge with $M=N-1$ piers, and we discuss separately the cases when the number $M$ of the piers is either odd or even. All buckled solutions are determined in a closed form and belong to $C^{2}(0,1)$. Each of them is constructed by rescaling and suitably collecting positive and negative solutions, $u_{(1)}^{+}$and $u_{(1)}^{-}$. For any given $N$, a general explicit formula is established to compute the bifurcation values as a function of $k$.

\section{Stationary states I}

\subsection{A single span without piers $(N=1)$}

The set $\mathcal{S}_{\kappa}$ of the bridge equilibria in this case consists of all (weak) solutions $u_{(1)}$ to the following boundary value problem on the interval $[0,1]$ (see Eq. (1.1))

$$
\left\{\begin{array}{l}
u^{\prime \prime \prime \prime}+\left(b \pi^{2}-\left\|u^{\prime}\right\|_{L^{2}(0,1)}^{2}\right) u^{\prime \prime}+\kappa \pi^{4} u^{+}=0 \\
u(0)=u(1)=u^{\prime \prime}(0)=u^{\prime \prime}(1)=0
\end{array}\right.
$$

It is worth noting that $\mathcal{S}_{\kappa}$ is bounded in $H^{2}(0,1) \cap H_{0}^{1}(0,1)$ for all $b \in \mathbb{R}, \kappa \in \mathbb{R}^{+}$(see [20]).

When $\kappa=0$, a general result was established in [7] for a class of non-vanishing sources. In [4], the same strategy with minor modifications was applied to a problem close to (2.1), where the one-sided springs are replaced by unyielding ties. We summarize here the results concerning stationary solutions in the case $\kappa=0$.

Theorem 2.1 (see [7], Th. 4.1) When $\kappa$ vanishes, the set $\mathcal{S}_{0}$ is finite for all $b \in \mathbb{R}$. Letting $\mathbb{S}_{b}=\left\{j \in \mathbb{N}: b>j^{2}\right\}$ and $j_{*}=\left|\mathbb{S}_{b}\right|, \mathcal{S}_{0}$ has exactly $2 j_{*}+1$ elements:

$$
u_{0}=0 \quad \text { and } \quad u_{j}^{ \pm}(x)=C_{j}^{ \pm} \sin j \pi x, \quad j \in \mathbb{S}_{b} \text {, }
$$



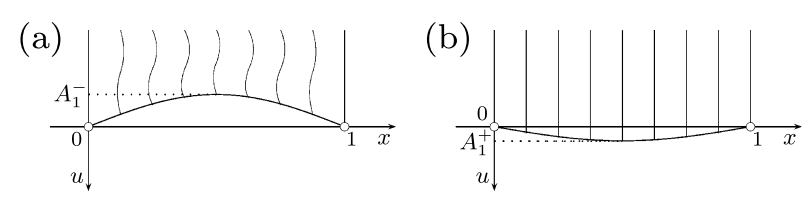

Figure 2 The buckled solutions $u_{(1)}^{-}$(a) and $u_{(1)}^{+}$(b) when $\kappa=0.1, b=1.125$.

where

$$
C_{j}^{ \pm}= \pm \frac{1}{j} \sqrt{2\left(b-j^{2}\right)}
$$

The general case is much more complicated. Since the scheme devised in $[5,7]$ does not work in the present situation, we obtain here a limited result.

Theorem 2.2 (Existence of buckled solutions) When $\kappa>0$, besides the null solution $u_{0}$, which exists for all $b \in \mathbb{R}$, problem (2.1) admits

- a negative buckled solution, $u_{(1)}^{-}$, if $b>1$;

- a positive buckled solution, $u_{(1)}^{+}$, if $b>1+\kappa$.

Proof For all values $b \in \mathbb{R}$, problem (2.1) has at least the trivial solution, $u_{0}=0$. For all $b>1$, besides the null solution, we obtain also the negative buckled solution

$$
u_{(1)}^{-}(x)=A_{1}^{-} \sin (\pi x), \quad \text { with } A_{1}^{-}\left(=C_{1}^{-}\right)=-\sqrt{2(b-1)},
$$

which solves the Woinowsky-Krieger problem (see [21])

$$
\left\{\begin{array}{l}
u^{\prime \prime \prime \prime}+\left(b \pi^{2}-\left\|u^{\prime}\right\|_{L^{2}(0,1)}^{2}\right) u^{\prime \prime}=0, \quad \text { in }[0,1] \\
u(0)=u(1)=u^{\prime \prime}(0)=u^{\prime \prime}(1)=0 .
\end{array}\right.
$$

If $b \leq 1$, there exists only the null solution. Therefore, $b=1$ will be referred to as the smallest bifurcation value. By paralleling [5], we infer that when $b>1+\kappa$, system (2.1) admits the positive buckled solution

$$
u_{(1)}^{+}(x)=A_{1}^{+} \sin (\pi x), \quad \text { with } A_{1}^{+}=\sqrt{2(b-1-\kappa)} .
$$

Since $\kappa>0$, we can easily check that $u_{(1)}^{+}(x)<\left|u_{(1)}^{-}(x)\right|$ for all $x \in(0,1)$ (see Figure 2 ).

\subsection{A bridge with a single pier $(N=2)$}

Now we consider a bridge with a single pier at $x=x_{1}$ and two sub-spans. The whole solution $u_{(2)}=\left(u_{1}, u_{2}\right)$ is obtained by joining their deflections, $u_{1}$ and $u_{2}$, which solve problems (1.2) for $n=1,2$ and fulfill the mutual groove condition

$$
u_{1}\left(x_{1}\right)=u_{2}^{\prime}\left(x_{1}\right)
$$

When $\left|I_{1}\right|=\left|I_{2}\right|$, it is easy to check that this condition cannot be satisfied by any buckled solution. Then we choose $\left|I_{1}\right| \neq\left|I_{2}\right|$. Let $x_{1}=\varepsilon$ be the point at which the pier is located, so that $\left|I_{1}\right|=\varepsilon$. For the sake of definiteness, let $u_{1}>0$ and $u_{2}<0$. 
Theorem 2.3 When $\kappa>0$, problems (1.2) for $N=2$ admit two buckled solutions, called $u_{(2)}^{+}$and $u_{(2)}^{-}$, provided that $\varepsilon=\varepsilon_{\kappa}$ and $b>b_{\kappa}$, where the values of $\varepsilon_{\kappa}$ and $b_{\kappa}$ are defined in (2.9) and (2.10), respectively.

Proof In order to establish the form of $u_{1}$ and $u_{2}$, we rescale the domains $[0, \varepsilon]$ and $[\varepsilon, 1]$ by virtue of the transformations $x=\varepsilon y$ and $x=(1-\varepsilon) z+\varepsilon$, respectively. Hence, we get the problem

$$
\begin{cases}w^{\prime \prime \prime \prime}+\varepsilon^{2}\left(b \pi^{2}-\frac{1}{\varepsilon^{2}}\left\|w^{\prime}\right\|_{L^{2}(0,1)}^{2}\right) w^{\prime \prime}+\varepsilon^{4} \kappa \pi^{4} w=0, & \text { in }[0,1] \\ v^{\prime \prime \prime \prime}+(1-\varepsilon)^{2}\left(b \pi^{2}-\frac{1}{(1-\varepsilon)^{2}}\left\|v^{\prime}\right\|_{L^{2}(0,1)}^{2}\right) v^{\prime \prime}=0, & \text { in }[0,1], \\ w(0)=w(1)=w^{\prime \prime}(0)=w^{\prime \prime}(1)=0, & \\ v(0)=v(1)=v^{\prime \prime}(0)=v^{\prime \prime}(1)=0, & \end{cases}
$$

where $w(y)=u_{1}(\varepsilon y)$ and $v(z)=u_{2}([1-\varepsilon] z+\varepsilon)$. Obviously, the null solution $v=w=0$ exists for all $b \in \mathbb{R}$. On the contrary, nontrivial solutions occur under special conditions.

Arguing as in Section 2.1, we exploit the explicit expression of solutions $u_{(1)}^{+}$and $u_{(1)}^{-}$, respectively, and we obtain

$$
w(y)=A_{2}^{+}(\varepsilon) \sin (\pi y) \quad \text { and } \quad v(z)=A_{2}^{-}(\varepsilon) \sin (\pi z)
$$

where, for some $\varepsilon_{\kappa} \in(0,1)$,

$$
A_{2}^{+}\left(\varepsilon_{\kappa}\right)=\varepsilon_{\kappa} \sqrt{2\left[b-\frac{1}{\varepsilon_{\kappa}^{2}}-\kappa \varepsilon_{\kappa}^{2}\right]}, \quad A_{2}^{-}\left(\varepsilon_{\kappa}\right)=\left(\varepsilon_{\kappa}-1\right) \sqrt{2\left[b-\frac{1}{\left(1-\varepsilon_{\kappa}\right)^{2}}\right]} .
$$

Of course, such solutions both exist provided that

$$
b>b_{\kappa}=\max \left\{\frac{1}{\left(1-\varepsilon_{\kappa}\right)^{2}}, \frac{1+\kappa \varepsilon_{\kappa}^{4}}{\varepsilon_{\kappa}^{2}}\right\}
$$

and $\varepsilon_{\kappa}$ satisfies the continuity condition (2.3), namely

$$
\frac{w^{\prime}(1)}{\varepsilon_{\kappa}}=\frac{v^{\prime}(0)}{1-\varepsilon_{\kappa}} .
$$

Then, by joining $w$ and $v$, we obtain the whole solution

$$
u_{(2)}^{+}(x)= \begin{cases}A_{2}^{+}\left(\varepsilon_{\kappa}\right) \sin \left(\pi x / \varepsilon_{\kappa}\right), & x \in\left[0, \varepsilon_{\kappa}\right), \\ 0, & x=\varepsilon_{\kappa}, \\ A_{2}^{-}\left(\varepsilon_{\kappa}\right) \sin \left(\pi\left(x-\varepsilon_{\kappa}\right) /\left(1-\varepsilon_{\kappa}\right)\right), & x \in\left(\varepsilon_{\kappa}, 1\right] .\end{cases}
$$

The function $u_{(2)}^{-}=\left(u_{2}, u_{1}\right)$, which is obtained by symmetrizing $u_{(2)}^{+}$with respect to the vertical line $x=1 / 2$, solves (1.2) under the same conditions (see Figure 3 ). Its expression is given by replacing $\varepsilon_{\kappa}$ with $1-\varepsilon_{\kappa}$ and $A_{2}^{+}$with $A_{2}^{-}$into (2.6),

$$
u_{(2)}^{-}(x)= \begin{cases}A_{2}^{-}\left(\varepsilon_{\kappa}\right) \sin \left(\pi x /\left(1-\varepsilon_{\kappa}\right)\right), & x \in\left[0,1-\varepsilon_{\kappa}\right), \\ 0, & x=1-\varepsilon_{\kappa}, \\ A_{2}^{+}\left(\varepsilon_{\kappa}\right) \sin \left(\pi\left(x-1+\varepsilon_{\kappa}\right) / \varepsilon_{\kappa}\right), & x \in\left(1-\varepsilon_{\kappa}, 1\right] .\end{cases}
$$



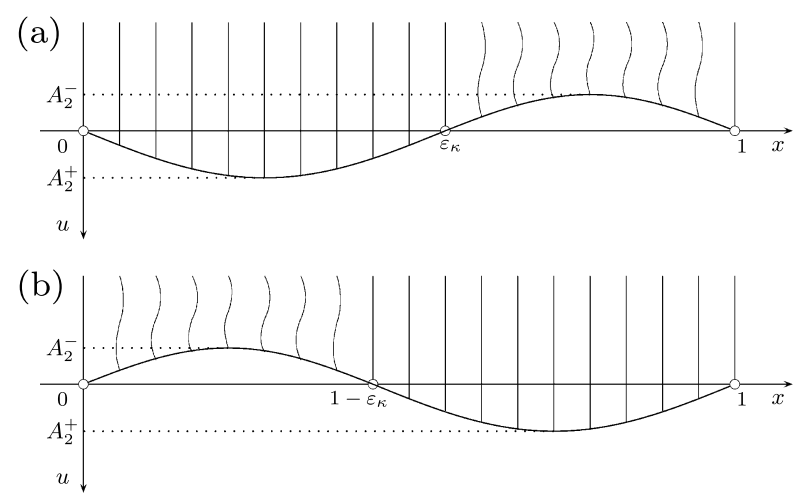

Figure 3 The buckled solutions $u_{(2)}^{+}$(a) and $u_{(2)}^{-}$(b) when $\kappa=5.9, b=5.75$.

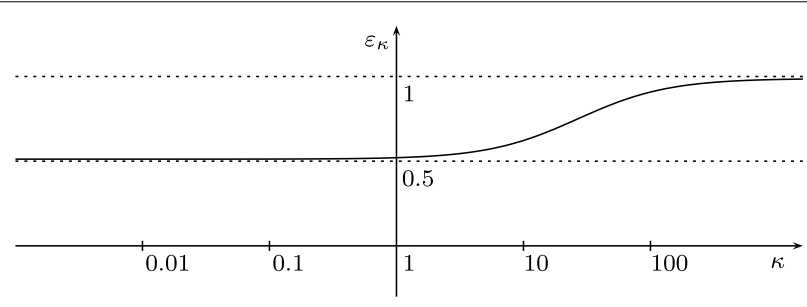

Figure 4 The value of $\varepsilon_{\kappa}$ as a function of $\kappa$.

Computation of $\varepsilon_{\kappa}$ and $b_{\kappa}$. For each $\kappa>0$, in order to compute the unknown value $\varepsilon_{\kappa}$, which ensures the existence of buckled solutions, we exploit the continuity condition (2.5), namely

$$
\left(1-\varepsilon_{\kappa}\right) A_{2}^{+}\left(\varepsilon_{\kappa}\right)+\varepsilon_{\kappa} A_{2}^{-}\left(\varepsilon_{\kappa}\right)=0 .
$$

Thus, the required value $\varepsilon_{\kappa}$ has to satisfy the system

$$
\left\{\begin{array}{l}
\frac{1}{\varepsilon_{\kappa}^{2}}+\kappa \varepsilon_{\kappa}^{2}=\frac{1}{\left(1-\varepsilon_{\kappa}\right)^{2}}, \\
0<\varepsilon_{\kappa}<1,
\end{array}\right.
$$

which implies

$$
\kappa=\frac{2 \varepsilon_{\kappa}-1}{\varepsilon_{\kappa}^{4}\left(1-\varepsilon_{\kappa}\right)^{2}} .
$$

It can be easily checked (see Figure 4$)$ that $\varepsilon_{\kappa} \in(1 / 2,1)$ and

$$
\lim _{\kappa \rightarrow 0} \varepsilon_{\kappa} \rightarrow \varepsilon_{0}=\frac{1}{2}, \quad \lim _{\kappa \rightarrow+\infty} \varepsilon_{\kappa}=1 .
$$

In order to compute the critical value of the axial force, $b_{\kappa}$, we observe that by virtue of (2.9), we have

$$
b_{\kappa}=\frac{1}{\left(1-\varepsilon_{\kappa}\right)^{2}}=\frac{1+\kappa \varepsilon_{\kappa}^{4}}{\varepsilon_{\kappa}^{2}} .
$$


Summarizing, for any given $\kappa>0$, if $b \leq b_{\kappa}$ no buckled solution exists, if $b>b_{\kappa}$ there exists a unique value of $\varepsilon_{\kappa}$, which produces two buckled solutions. As a consequence, $b_{\kappa}$ represents the bifurcation value. According to (2.10), we get

$$
A_{2}^{+}\left(\varepsilon_{\kappa}\right)=\varepsilon_{\kappa} \sqrt{2\left(b-b_{\kappa}\right)}, \quad A_{2}^{-}\left(\varepsilon_{\kappa}\right)=\left(\varepsilon_{\kappa}-1\right) \sqrt{2\left(b-b_{\kappa}\right)}
$$

and $-A_{2}^{-}<A_{2}^{+}$. In addition, for all $\kappa>0$, we can easily obtain

$$
b_{\kappa}>b_{0}=4
$$

Then, when $b \leq 4$, the set $\mathcal{S}_{\kappa}$ contains only the trivial solution $u_{0}$.

In the limit $\kappa \rightarrow 0$, it follows that

$$
-A_{2}^{-}, A_{2}^{+} \rightarrow \sqrt{(b-4) / 2} \text {. }
$$

This means that solutions $u_{(2)}^{+}$and $u_{(2)}^{-}$tend to coincide with the second bifurcation branch of problem (2.2), as expected.

\subsection{A bridge with two piers $(N=3)$}

When the bridge has two intermediate piers and three sub-spans, we shall construct solutions $u_{(3)}=\left(u_{1}, u_{2}, u_{3}\right)$, where $u_{1}, u_{2}$ and $u_{3}$ solve (1.2). It is easy to check that no buckled solution exists when $\left|I_{1}\right|=\left|I_{2}\right|=\left|I_{3}\right|$. Then we choose $\left|I_{1}\right|=\left|I_{3}\right| \neq\left|I_{2}\right|$ and construct a buckled solution by joining three (suitably rescaled) functions which have the form of either $u_{(1)}^{+}$or $u_{(1)}^{-}$.

Theorem 2.4 When $\kappa>0$, problems (1.2) for $N=3$ admit two buckled solutions, $u_{(3)}^{+}$and $u_{(3)}^{-}$, provided that $b>c_{\kappa}^{+}$and $b>c_{\kappa}^{-}$, respectively, where the values of $c_{\kappa}^{+}$and $c_{\kappa}^{-}$are defined in (2.12) and (2.15).

Proof The buckled solutions are constructed as follows. The former, $u_{(3)}^{+}$, has two positive components with the same shape on $I_{1}$ and $I_{3}$, and a negative one on $I_{2}$. The latter, $u_{(3)}^{-}$, is negative in $I_{1}$ and $I_{3}$, but positive in $I_{2}$. Accordingly, let

$$
\begin{aligned}
& w_{j}=\left.u_{(3)}^{+}\right|_{2 j-1}, \quad j=1,2 \quad \text { and } \quad v_{0}=\left.u_{(3)}^{+}\right|_{I_{2}}, \\
& v_{j}=\left.u_{(3)}^{-}\right|_{2 j-1}, \quad j=1,2 \quad \text { and } \quad w_{0}=\left.u_{(3)}^{-}\right|_{I_{2}},
\end{aligned}
$$

so that $w_{k}>0$ and $v_{k}<0$ for $k=0,1,2$.

In order to construct $u_{(3)}^{+}$, we exploit the same argument as in Section 2.2. First, we argue that

$$
\left|I_{1}\right|=\left|I_{3}\right|=\xi, \quad\left|I_{2}\right|=1-2 \xi, \quad \xi \in(0,1 / 2) .
$$


Then, we apply the rescaling procedure with the scale factor $\xi /(1-\xi)=\varepsilon$ and, by virtue of the transformations $x=\xi y$ and $x=(1-2 \xi) z+\xi$, we obtain

$$
\begin{cases}w_{j}^{\prime \prime \prime \prime}+\xi^{2}\left(b \pi^{2}-\frac{1}{\xi^{2}}\left\|w_{j}^{\prime}\right\|_{L^{2}(0,1)}^{2}\right) w_{j}^{\prime \prime}+\kappa \xi^{4} \pi^{4} w_{j}=0, & \text { in }[0,1], j=1,2 \\ v_{0}^{\prime \prime \prime \prime}+(1-2 \xi)^{2}\left(b \pi^{2}-\frac{1}{(1-2 \xi)^{2}}\left\|v_{0}^{\prime}\right\|_{L^{2}(0,1)}^{2}\right) v_{0}^{\prime \prime}=0, & \text { in }[0,1], \\ w_{j}(0)=w_{j}(1)=w_{j}^{\prime \prime}(0)=w_{j}^{\prime \prime}(1)=0, & j=1,2, \\ v_{0}(0)=v_{0}(1)=v_{0}^{\prime \prime}(0)=v_{0}^{\prime \prime}(1)=0 . & \end{cases}
$$

Taking into account that $\varepsilon=\varepsilon_{\kappa}$ is uniquely and implicitly given by (2.9), the corresponding value of $\xi$ becomes

$$
\xi_{\kappa}=\frac{\varepsilon_{\kappa}}{1+\varepsilon_{\kappa}}
$$

so that

$$
\lim _{\kappa \rightarrow 0} \xi_{\kappa}=\xi_{0}=\frac{1}{3}, \quad \lim _{\kappa \rightarrow+\infty} \xi_{\kappa}=\frac{1}{2} .
$$

Accordingly, we obtain

$$
w_{j}(y)=A_{3}^{+}\left(\xi_{\kappa}\right) \sin (\pi y), \quad j=1,2, \quad v_{0}(z)=A_{3}^{-}\left(\xi_{\kappa}\right) \sin (\pi z),
$$

where

$$
A_{3}^{+}\left(\xi_{\kappa}\right)=\xi_{\kappa} \sqrt{2\left[b-\frac{1}{\xi_{\kappa}^{2}}-\kappa \xi_{\kappa}^{2}\right]}, \quad A_{3}^{-}\left(\xi_{\kappa}\right)=\left(2 \xi_{\kappa}-1\right) \sqrt{2\left[b-\frac{1}{\left(1-2 \xi_{\kappa}\right)^{2}}\right]} .
$$

Of course, such solutions exist at the same time, provided that $b>c_{\kappa}^{+}$, where

$$
c_{\kappa}^{+}=\max \left\{\frac{1}{\left(1-2 \xi_{\kappa}\right)^{2}}, \frac{1+\kappa \xi_{\kappa}^{4}}{\xi_{\kappa}^{2}}\right\}=\max \left\{\frac{\left(1+\varepsilon_{\kappa}\right)^{2}}{\left(1-\varepsilon_{\kappa}\right)^{2}}, \frac{\left(1+\varepsilon_{\kappa}\right)^{4}+\kappa \varepsilon_{\kappa}^{4}}{\varepsilon_{\kappa}^{2}\left(1+\varepsilon_{\kappa}\right)^{2}}\right\},
$$

where $c_{\kappa}^{+}$represents the bifurcation value. By virtue of (2.9), we get

$$
c_{\kappa}^{+}=\frac{\left(1+\varepsilon_{\kappa}\right)^{2}}{\left(1-\varepsilon_{\kappa}\right)^{2}} .
$$

If this is the case, we have the whole solution (see Figure 5a)

$$
u_{(3)}^{+}(x)= \begin{cases}A_{3}^{+}\left(\xi_{\kappa}\right) \sin \left(\pi x / \xi_{\kappa}\right), & x \in\left[0, \xi_{\kappa}\right], \\ A_{3}^{-}\left(\xi_{\kappa}\right) \sin \left(\pi\left(x-\xi_{\kappa}\right) /\left(1-2 \xi_{\kappa}\right)\right), & x \in\left[\xi_{\kappa}, 1-\xi_{\kappa}\right], \\ A_{3}^{+}\left(\xi_{\kappa}\right) \sin \left(\pi\left(x-1+\xi_{\kappa}\right) / \xi_{\kappa}\right), & x \in\left[1-\xi_{\kappa}, 1\right] .\end{cases}
$$

In order to construct $u_{(3)}^{-}$, we proceed as before by letting

$$
\left|I_{1}\right|=\left|I_{3}\right|=\eta, \quad\left|I_{2}\right|=1-2 \eta, \quad \eta \in(0,1 / 2) .
$$




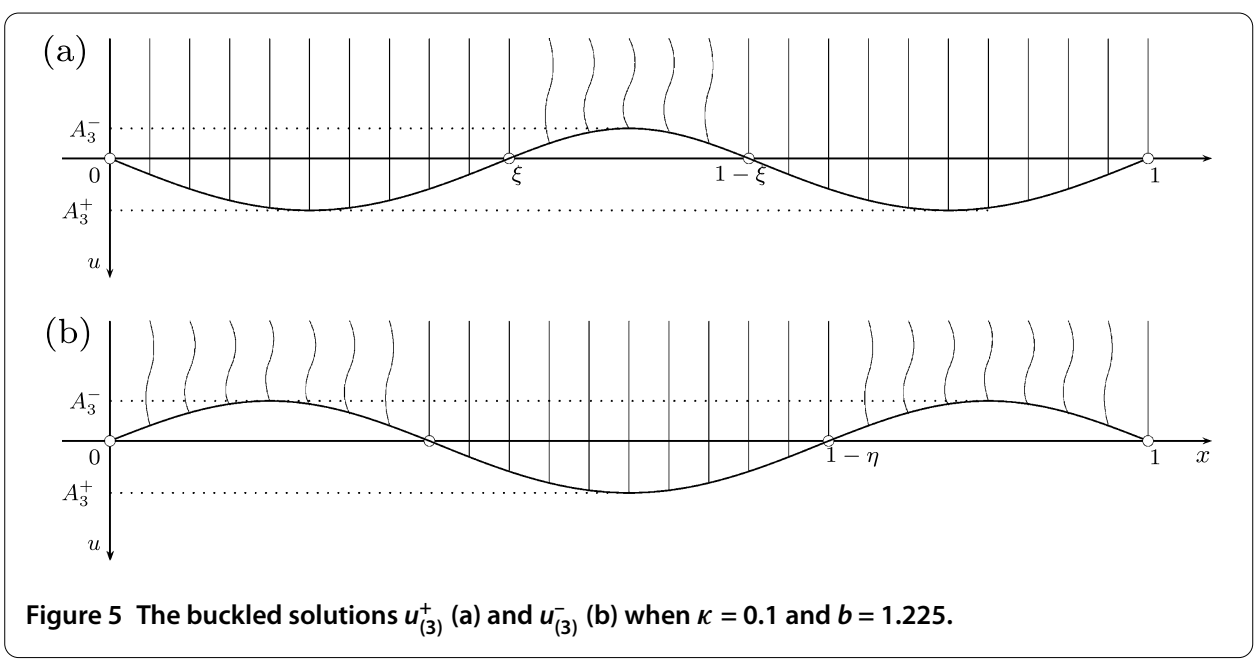

By means of a rescaling procedure with the scale factor $\eta /(1-\eta)=1-\varepsilon$, the transformations $x=\eta z$ and $x=(1-2 \eta) y+\eta$ lead to

$$
\begin{cases}v_{j}^{\prime \prime \prime \prime}+\eta^{2}\left(b \pi^{2}-\frac{1}{\eta^{2}}\left\|v_{j}^{\prime}\right\|_{L^{2}(0,1)}^{2}\right) v_{j}^{\prime \prime}=0, & \text { in }[0,1], j=1,2, \\ w_{0}^{\prime \prime \prime \prime}+(1-2 \eta)^{2}\left(b \pi^{2}-\frac{1}{(1-2 \eta)^{2}}\left\|w_{0}^{\prime}\right\|_{L^{2}(0,1)}^{2}\right) w_{0}^{\prime \prime} & \\ \quad+\kappa(1-2 \eta)^{4} \pi^{4} w_{0}=0, & \text { in }[0,1], \\ v_{j}(0)=v_{j}(1)=v_{j}^{\prime \prime}(0)=v_{j}^{\prime \prime}(1)=0, & j=1,2, \\ w_{0}(0)=w_{0}(1)=w_{0}^{\prime \prime}(0)=w_{0}^{\prime \prime}(1)=0 . & \end{cases}
$$

Accordingly, we find

$$
\eta_{\kappa}=\frac{1-\varepsilon_{\kappa}}{2-\varepsilon_{\kappa}}
$$

where $\varepsilon_{\kappa}$ is given by (2.9), and

$$
\lim _{\kappa \rightarrow 0} \eta_{\kappa}=\eta_{0}=\frac{1}{3}, \quad \lim _{\kappa \rightarrow+\infty} \eta_{\kappa}=0 .
$$

So, we obtain

$$
v_{j}(z)=A_{3}^{-}\left(\eta_{\kappa}\right) \sin (\pi z), \quad j=1,2, \quad w_{0}(y)=A_{3}^{+}\left(\eta_{\kappa}\right) \sin (\pi y),
$$

where

$$
\begin{aligned}
& A_{3}^{-}\left(\eta_{\kappa}\right)=-\eta_{\kappa} \sqrt{2\left[b-\frac{1}{\eta_{\kappa}^{2}}\right]}, \\
& A_{3}^{+}\left(\eta_{\kappa}\right)=\left(1-2 \eta_{\kappa}\right) \sqrt{2\left[b-\frac{1}{\left(1-2 \eta_{\kappa}\right)^{2}}-\kappa\left(1-2 \eta_{\kappa}\right)^{2}\right]} .
\end{aligned}
$$

Of course, such solutions do exist together provided that

$$
b>c_{\kappa}^{-}=\max \left\{\frac{1}{\eta_{\kappa}^{2}}, \frac{1+\kappa\left(1-2 \eta_{\kappa}\right)^{4}}{\left(1-2 \eta_{\kappa}\right)^{2}}\right\}=\max \left\{\frac{\left(2-\varepsilon_{\kappa}\right)^{2}}{\left(1-\varepsilon_{\kappa}\right)^{2}}, \frac{\left(2-\varepsilon_{\kappa}\right)^{4}+\kappa \varepsilon_{\kappa}^{4}}{\varepsilon_{\kappa}^{2}\left(2-\varepsilon_{\kappa}\right)^{2}}\right\},
$$


where $c_{\kappa}^{-}$represents the bifurcation value. By virtue of (2.9), we get

$$
c_{\kappa}^{-}=\frac{\left(2-\varepsilon_{\kappa}\right)^{2}}{\left(1-\varepsilon_{\kappa}\right)^{2}} .
$$

By means of (2.9) it is easy to check that $c_{\kappa}^{-}<c_{\kappa}^{+}$. The complete solution is given by (see Figure 5b)

$$
u_{(3)}^{-}(x)= \begin{cases}A_{3}^{-}\left(\eta_{\kappa}\right) \sin \left(\pi x / \eta_{\kappa}\right), & x \in\left[0, \eta_{\kappa}\right], \\ A_{3}^{+}\left(\eta_{\kappa}\right) \sin \left(\pi\left(x-\eta_{\kappa}\right) /\left(1-2 \eta_{\kappa}\right)\right), & x \in\left[\eta_{\kappa}, 1-\eta_{\kappa}\right], \\ A_{3}^{-}\left(\eta_{\kappa}\right) \sin \left(\pi\left(x-1+\eta_{\kappa}\right) / \eta_{\kappa}\right), & x \in\left[1-\eta_{\kappa}, 1\right] .\end{cases}
$$

\section{Stationary states II}

In this section we generalize the problem to a bridge with $N$ sub-spans and $M=N-1$ piers. The existence of buckled solutions is investigated in connection with the length of the sub-spans. Indeed, it is easy to check that no buckled solution exists when all of them are of the same length. Then a buckled solution may be obtained by collecting and joining $N$ (suitably rescaled) functions of the same form as either $u_{(1)}^{+}$or $u_{(1)}^{-}$. To this end, we are forced to consider separately the cases when the number $M$ of the piers is either odd or even. In the former case, indeed, we adopt a strategy which is close to that applied in Section 2.2. In the latter, we iterate the procedure devised in Section 2.3.

Theorem 3.1 For any $\kappa>0, N \in \mathbb{N}$, (1.2) admits two buckled solutions.

- In the odd case, $M=2 m-1$, there exist $u_{(2 m)}^{+}$and $u_{(2 m)}^{-}$provided that $b>b_{\kappa}(m)$, where the value of $b_{\kappa}(m)$ is characterized in (3.5).

- In the even case, $M=2 m$, there exist $u_{(2 m+1)}^{+}$and $u_{(2 m+1)}^{-}$provided that $b>c_{\kappa}^{+}(m)$ and $b>c_{\kappa}^{-}(m)$, respectively, where the values of $c_{\kappa}^{+}(m)$ and $c_{\kappa}^{-}(m)$ are characterized in (3.6).

Proof The odd case. Solutions $u_{(2 m)}^{+}$and $u_{(2 m)}^{-}, m=(M+1) / 2=N / 2$, are assumed to change the sign alternately on the sub-spans. The superscript $+(-)$ means that the solution is positive (negative) on $I_{1}$. In order to construct them, we join $2 m$ rescaled functions of the same form as either $u_{(1)}^{+}$or $u_{(1)}^{-}$. Arguing as in the step $N=2$, we let

$$
\left|I_{2 j-1}\right|=\frac{\varepsilon_{\kappa}}{m}, \quad j=1, \ldots, m, \quad\left|I_{2 h+2}\right|=\frac{1-\varepsilon_{\kappa}}{m}, \quad h=0, \ldots, m-1,
$$

where $\varepsilon_{\kappa}$ is given by (2.9).

CONSTRUCTION OF $u_{(2 m)}^{+}$. Let

$$
w_{j}=\left.u_{(2 m)}^{+}\right|_{2 j-1}, \quad j=1, \ldots, m, \quad v_{h}=\left.u_{(2 m)}^{+}\right|_{2 h+2}, \quad h=0, \ldots, m-1,
$$

so that $w_{j}>0$ and $v_{h}<0$. Since

$$
\left|I_{2 j-1} \cup I_{2 j}\right|=\frac{1}{m},
$$

we stress that each restriction

$$
\left.u_{(2 m)}^{+}\right|_{I_{2 j-1} \cup I_{2 j}}, \quad j=1, \ldots, m,
$$


is similar to $u_{(2)}^{+}$, rescaled by a factor $1 / m$. In particular,

$$
\begin{aligned}
& I_{2 j-1}=\left(\frac{j-1}{m}, \frac{j-1+\varepsilon_{\kappa}}{m}\right), \quad j=1, \ldots, m, \\
& I_{2 h+2}=\left(\frac{h+\varepsilon_{\kappa}}{m}, \frac{h+1}{m}\right), \quad h=0, \ldots, m-1 .
\end{aligned}
$$

Therefore, on $I_{2 j-1}$ we have

$$
w_{j}(x)=A_{2 m}^{+} \sin \left(\pi\left(x-\frac{j-1}{m}\right) / \frac{\varepsilon_{\kappa}}{m}\right), \quad j=1, \ldots, m,
$$

while on $I_{2 h+2}$ we obtain

$$
v_{h}(x)=A_{2 m}^{-} \sin \left(\pi\left(x-\frac{h+\varepsilon_{\kappa}}{m}\right) / \frac{1-\varepsilon_{\kappa}}{m}\right), \quad h=0, \ldots, m-1,
$$

where

$$
A_{2 m}^{+}=\frac{\varepsilon_{\kappa}}{m} \sqrt{2\left[b-\frac{m^{2}}{\varepsilon_{\kappa}{ }^{2}}-\frac{\kappa \varepsilon_{\kappa}{ }^{2}}{m^{2}}\right]}, \quad A_{2 m}^{-}=\frac{\varepsilon_{\kappa}-1}{m} \sqrt{2\left[b-\frac{m^{2}}{\left(1-\varepsilon_{\kappa}\right)^{2}}\right]} .
$$

Such solutions exist provided that

$$
b>b_{\kappa}(m)=\max \left\{\frac{m^{2}}{\varepsilon_{\kappa}{ }^{2}}+\frac{\kappa \varepsilon_{\kappa}^{2}}{m^{2}}, \frac{m^{2}}{\left(1-\varepsilon_{\kappa}\right)^{2}}\right\} .
$$

By virtue of Lemma 3.2, which will be proved later, we get

$$
b_{\kappa}(m)=m^{2} b_{\kappa}, \quad m=N / 2 .
$$

\section{Construction of $u_{(2 m)}^{-}$. Let}

$$
v_{j}=\left.u_{(2 m)}^{-}\right|_{2 j-1}, j=1, \ldots, m, \quad w_{h}=u_{(2 m)}^{-} \mid I_{2 h+2}, \quad h=0, \ldots, m-1
$$

so that $w_{h}>0$ and $v_{j}<0$. Arguing as before, we obtain

$$
\left|I_{2 j-1}\right|=\frac{1-\varepsilon_{\kappa}}{m}, \quad j=1, \ldots, m, \quad\left|I_{2 h+2}\right|=\frac{\varepsilon_{\kappa}}{m}, \quad h=0, \ldots, m-1,
$$

where $\varepsilon_{\kappa}$ is given by $(2.9)$ and

$$
\left.u_{(2 m)}^{-}\right|_{I_{2 j-1} \cup I_{2 j}}, \quad j=1, \ldots, m,
$$

is similar to $u_{(2)}^{-}$, rescaled by a factor $1 / m$. In particular,

$$
\begin{aligned}
& I_{2 j-1}=\left(\frac{j-1}{m}, \frac{j-\varepsilon_{\kappa}}{m}\right), \quad j=1, \ldots, m, \\
& I_{2 h+2}=\left(\frac{h+1-\varepsilon_{\kappa}}{m}, \frac{h+1}{m}\right), \quad h=0, \ldots, m-1,
\end{aligned}
$$


so on $I_{2 j-1}$ we have

$$
v_{j}(x)=A_{2 m}^{-} \sin \left(\pi\left(x-\frac{j-1}{m}\right) / \frac{1-\varepsilon_{\kappa}}{m}\right), \quad j=1, \ldots, m,
$$

while on $I_{2 h+2}$ we obtain

$$
w_{h}(x)=A_{2 m}^{+} \sin \left(\pi\left(x-\frac{h+1-\varepsilon_{\kappa}}{m}\right) / \frac{\varepsilon_{\kappa}}{m}\right), \quad h=0, \ldots, m-1,
$$

with $A_{2 m}^{+}, A_{2 m}^{-}$defined as in (3.1).

The even case. In this case, we construct the solutions $u_{(2 m+1)}^{+}$and $u_{(2 m+1)}^{-}$, where $m=$ $M / 2=(N-1) / 2$.

Construction of $u_{(2 m+1)}^{+}$. Let

$$
\begin{aligned}
& w_{j}=\left.u_{(2 m+1)}^{+}\right|_{I_{2 j-1}}, \quad j=1, \ldots, m+1, \\
& v_{h}=\left.u_{(2 m+1)}^{+}\right|_{I_{2 h+2}}, \quad h=0, \ldots, m-1,
\end{aligned}
$$

so that $w_{j}>0$ and $v_{h}<0$. Arguing as in the step $N=3$, we obtain

$$
\begin{aligned}
& \left|I_{2 j-1}\right|=\xi_{\kappa}, \quad j=1, \ldots, m+1, \\
& \left|I_{2 h+2}\right|=\frac{1-(1+m) \xi_{\kappa}}{m}, \quad h=0, \ldots, m-1,
\end{aligned}
$$

where $\xi_{\kappa}$ is given by $\xi_{\kappa}=\frac{\varepsilon_{\kappa}}{m+\varepsilon_{\kappa}}$. Therefore

$$
\begin{aligned}
& \left|I_{2 j-1}\right|=\frac{\varepsilon_{\kappa}}{m+\varepsilon_{\kappa}}, \\
& \left|I_{2 h+2}\right|=\frac{1-\varepsilon_{\kappa}}{m+\varepsilon_{\kappa}}
\end{aligned}
$$

and

$$
\begin{aligned}
& I_{2 j-1}=\left(\frac{j-1}{m+\varepsilon_{\kappa}}, \frac{j-1+\varepsilon_{\kappa}}{m+\varepsilon_{\kappa}}\right), \quad j=1, \ldots, m+1, \\
& I_{2 h+2}=\left(\frac{h+\varepsilon_{\kappa}}{m+\varepsilon_{\kappa}}, \frac{h+1}{m+\varepsilon_{\kappa}}\right), \quad h=0, \ldots, m-1 .
\end{aligned}
$$

So, on $I_{2 j-1}$ we have

$$
w_{j}(x)=A_{2 m+1}^{+} \sin \left(\pi\left(x-\frac{j-1}{m+\varepsilon_{\kappa}}\right) / \frac{\varepsilon_{\kappa}}{m+\varepsilon_{\kappa}}\right), \quad j=1, \ldots, m+1,
$$

while on $I_{2 h+2}$ we obtain

$$
v_{h}(x)=A_{2 m+1}^{-} \sin \left(\pi\left(x-\frac{h+\varepsilon_{\kappa}}{m+\varepsilon_{\kappa}}\right) / \frac{1-\varepsilon_{\kappa}}{m+\varepsilon_{\kappa}}\right), \quad h=0, \ldots, m-1,
$$


where

$$
\begin{aligned}
& A_{2 m+1}^{+}=\frac{\varepsilon_{\kappa}}{m+\varepsilon_{\kappa}} \sqrt{2\left[b-\frac{\left(m+\varepsilon_{\kappa}\right)^{2}}{\varepsilon_{\kappa}{ }^{2}}-\frac{\kappa \varepsilon_{\kappa}{ }^{2}}{\left(m+\varepsilon_{\kappa}\right)^{2}}\right]}, \\
& A_{2 m+1}^{-}=\frac{\varepsilon_{\kappa}-1}{m+\varepsilon_{\kappa}} \sqrt{2\left[b-\frac{\left(m+\varepsilon_{\kappa}\right)^{2}}{\left(1-\varepsilon_{\kappa}\right)^{2}}\right]} .
\end{aligned}
$$

Such solutions exist provided that

$$
b>c_{\kappa}^{+}(m)=\max \left\{\frac{\left(m+\varepsilon_{\kappa}\right)^{2}}{\varepsilon_{\kappa}^{2}}+\frac{\kappa \varepsilon_{\kappa}^{2}}{\left(m+\varepsilon_{\kappa}\right)^{2}}, \frac{\left(m+\varepsilon_{\kappa}\right)^{2}}{\left(1-\varepsilon_{\kappa}\right)^{2}}\right\} .
$$

By virtue of Lemma 3.2, we get

$$
c_{\kappa}^{+}(m)=\frac{\left(m+\varepsilon_{\kappa}\right)^{2}}{\left(1-\varepsilon_{\kappa}\right)^{2}}, \quad m=(N-1) / 2 .
$$

\section{Construction of $u_{(2 m+1)}^{-}$. Let}

$$
v_{j}=\left.u_{(2 m+1)}^{-}\right|_{2 j-1}, \quad j=1, \ldots, m+1, \quad w_{h}=\left.u_{(2 m+1)}^{-}\right|_{2 h+2}, \quad h=0, \ldots, m-1,
$$

so that $w_{h}>0$ and $v_{j}<0$. Arguing as in the step $N=3$, we obtain

$$
\begin{aligned}
& \left|I_{2 j-1}\right|=\eta_{\kappa}, \quad j=1, \ldots, m+1, \\
& \left|I_{2 h+2}\right|=\frac{1-(1+m) \eta_{\kappa}}{m}, \quad h=0, \ldots, m-1,
\end{aligned}
$$

where $\eta_{\kappa}$ is given by $\eta_{\kappa}=\frac{1-\varepsilon_{\kappa}}{m+1-\varepsilon_{\kappa}}$. Therefore

$$
\left|I_{2 j-1}\right|=\frac{1-\varepsilon_{\kappa}}{m+1-\varepsilon_{\kappa}}, \quad\left|I_{2 h+2}\right|=\frac{\varepsilon_{\kappa}}{m+1-\varepsilon_{\kappa}}
$$

and

$$
\begin{aligned}
& I_{2 j-1}=\left(\frac{j-1}{m+1-\varepsilon_{\kappa}}, \frac{j-\varepsilon_{\kappa}}{m+1-\varepsilon_{\kappa}}\right), \quad j=1, \ldots, m+1, \\
& I_{2 h+2}=\left(\frac{h+1-\varepsilon_{\kappa}}{m+1-\varepsilon_{\kappa}}, \frac{h+1}{m+1-\varepsilon_{\kappa}}\right), \quad h=0, \ldots, m-1 .
\end{aligned}
$$

As a consequence, on $I_{2 j-1}$ we have

$$
v_{j}(x)=B_{2 m+1}^{-} \sin \left(\pi\left(x-\frac{j-1}{m+1-\varepsilon_{\kappa}}\right) / \frac{1-\varepsilon_{\kappa}}{m+1-\varepsilon_{\kappa}}\right), \quad j=1, \ldots, m+1,
$$

while on $I_{2 h+2}$ we obtain

$$
w_{h}(x)=B_{2 m+1}^{+} \sin \left(\pi\left(x-\frac{h+1-\varepsilon_{\kappa}}{m+1-\varepsilon_{\kappa}}\right) / \frac{\varepsilon_{\kappa}}{m+1-\varepsilon_{\kappa}}\right), \quad h=0, \ldots, m-1,
$$


where

$$
\begin{aligned}
& B_{2 m+1}^{+}=\frac{\varepsilon_{\kappa}}{m+1-\varepsilon_{\kappa}} \sqrt{2\left[b-\frac{\left(m+1-\varepsilon_{\kappa}\right)^{2}}{\varepsilon_{\kappa}{ }^{2}}-\frac{\kappa \varepsilon_{\kappa}{ }^{2}}{\left(m+1-\varepsilon_{\kappa}\right)^{2}}\right]}, \\
& B_{2 m+1}^{-}=\frac{\varepsilon_{\kappa}-1}{m+1-\varepsilon_{\kappa}} \sqrt{2\left[b-\frac{\left(m+1-\varepsilon_{\kappa}\right)^{2}}{\left(1-\varepsilon_{\kappa}\right)^{2}}\right]} .
\end{aligned}
$$

Such solutions exist provided that $b>c_{\kappa}^{-}(m)$, where

$$
c_{\kappa}^{-}(m)=\max \left\{\frac{\left(m+1-\varepsilon_{\kappa}\right)^{2}}{\varepsilon_{\kappa}^{2}}+\frac{\kappa \varepsilon_{\kappa}^{2}}{\left(m+1-\varepsilon_{\kappa}\right)^{2}}, \frac{\left(m+1-\varepsilon_{\kappa}\right)^{2}}{\left(1-\varepsilon_{\kappa}\right)^{2}}\right\} .
$$

By virtue of Lemma 3.2, we get

$$
c_{\kappa}^{-}(m)=\frac{\left(m+1-\varepsilon_{\kappa}\right)^{2}}{\left(1-\varepsilon_{\kappa}\right)^{2}}, \quad m=(N-1) / 2
$$

Lemma 3.2 (Characterization of the bifurcation values) For any given $\kappa>0$ and $m \in \mathbb{N}$,

$$
\begin{aligned}
& b_{\kappa}(m)=\frac{m^{2}}{\left(1-\varepsilon_{\kappa}\right)^{2}}=m^{2} b_{\kappa}, \\
& c_{\kappa}^{-}(m)=\frac{\left(m+1-\varepsilon_{\kappa}\right)^{2}}{\left(1-\varepsilon_{\kappa}\right)^{2}}<c_{\kappa}^{+}(m)=\frac{\left(m+\varepsilon_{\kappa}\right)^{2}}{\left(1-\varepsilon_{\kappa}\right)^{2}},
\end{aligned}
$$

where $\varepsilon_{\kappa}$ and $b_{\kappa}$ are computed by (2.9) and (2.10), respectively.

Proof First, in view of (3.2), we have to prove that

$$
\frac{m^{2}}{\left(1-\varepsilon_{\kappa}\right)^{2}} \geq \frac{m^{4}+\kappa \varepsilon_{\kappa}^{4}}{\varepsilon_{\kappa}^{2}}
$$

which is equivalent to

$$
\kappa \leq m^{4} \frac{2 \varepsilon_{\kappa}-1}{\varepsilon_{\kappa}^{4}\left(1-\varepsilon_{\kappa}\right)^{2}} .
$$

By replacing the expression of $\kappa$ given by (2.9), we obtain

$$
1 \leq m^{4}
$$

which implies that (3.5) holds for all $m \in \mathbb{N}$. Then, in view of (3.4), we have to prove

$$
\frac{\left(m+1-\varepsilon_{\kappa}\right)^{2}}{\left(1-\varepsilon_{\kappa}\right)^{2}} \geq \frac{\left(m+1-\varepsilon_{\kappa}\right)^{4}+\kappa \varepsilon_{\kappa}^{4}}{\varepsilon_{\kappa}\left(m+1-\varepsilon_{\kappa}\right)^{2}},
$$

which is equivalent to

$$
\kappa \leq \frac{\left(2 \varepsilon_{\kappa}-1\right)\left(m+1-\varepsilon_{\kappa}\right)^{4}}{\varepsilon_{\kappa}^{4}\left(1-\varepsilon_{\kappa}\right)^{2}} .
$$



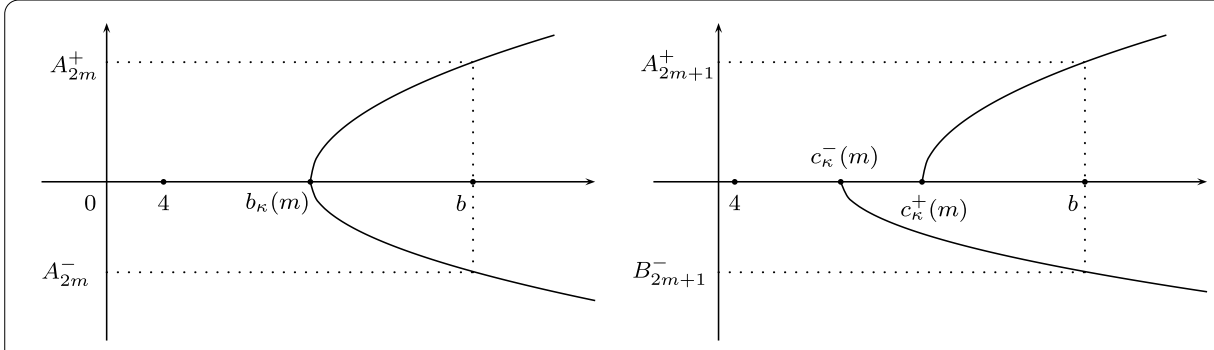

Figure 6 The bifurcation portrait: for $N$ odd (on the left, $m=N / 2$ ) and $N$ even (on the right, $m=(N-1) / 2)$.

By virtue of (2.9), we obtain

$$
1 \leq\left(m+1-\varepsilon_{\kappa}\right)^{4},
$$

which is identically satisfied for all admissible values $\varepsilon_{\kappa}$ and for all $m \in \mathbb{N}$. Hence, (3.6) holds. Finally, in view of (3.3), we need to prove

$$
\frac{\left(m+\varepsilon_{\kappa}\right)^{2}}{\left(1-\varepsilon_{\kappa}\right)^{2}} \geq \frac{\left(m+\varepsilon_{\kappa}\right)^{4}+\kappa \varepsilon_{\kappa}^{4}}{\varepsilon_{\kappa}\left(m+\varepsilon_{\kappa}\right)^{2}},
$$

and this is equivalent to

$$
\kappa \leq \frac{\left(2 \varepsilon_{\kappa}-1\right)\left(m+\varepsilon_{\kappa}\right)^{4}}{\varepsilon_{\kappa}^{4}\left(1-\varepsilon_{\kappa}\right)^{2}} .
$$

Applying (2.9) as in the previous cases, we obtain $1 \leq\left(m+\varepsilon_{\kappa}\right)^{4}$, which is identically satisfied for all admissible values $\varepsilon_{\kappa}$ and for all $m \in \mathbb{N}$ so that (3.6) $)_{2}$ follows. Moreover, starting from (3.6) and taking into account that $1 / 2<\varepsilon_{\kappa}<1$, it is easy to check that

$$
c_{\kappa}^{-}(m)<c_{\kappa}^{+}(m)
$$

Previous results can be summarized by means of a simple sketch which highlights the main bifurcation features (see Figure 6).

Finally, it is worth noting that by removing the coupling between the road-bed and the cable, we recover well-known results (see, for instance, $[5,8]$ )

Remark 3.3 In the limit $\kappa \rightarrow 0$, from (2.9) it follows $\varepsilon_{\kappa} \rightarrow 1 / 2$, so that buckled solutions exist even if all sub-spans are equal. Moreover,

$$
b_{\kappa}(m) \rightarrow(2 m)^{2}=N^{2}, \quad c_{\kappa}^{-}(m), c_{\kappa}^{+}(m) \rightarrow(2 m+1)^{2}=N^{2} .
$$

Competing interests

The authors declare that they have no competing interests.

\section{Authors' contributions}

CG conceived the study, participated in its design, verified all calculations and drew all figures, EV participated in the design of the study, performed the proofs of theorems and lemmas, carried out all calculations and drafted the manuscript. 


\section{References}

1. Bochicchio, I, Giorgi, C, Vuk, E: On some nonlinear models for suspension bridges. In: Andreucci, D, Carillo, S, Fabrizio, M, Loreti, P, Sforza, D (eds.) Evolution Equations and Materials with Memory, Proceedings, Rome 2010, pp. 1-17. Università La Sapienza Ed., Roma (2012)

2. Choo, SM, Chung, SK: Finite difference approximate solutions for the strongly damped extensible beam equations. Appl. Math. Comput. 112, 11-32 (2000)

3. Fang, W, Wickert, JA: Postbuckling of micromachined beams. J. Micromech. Microeng. 4, 116-122 (1994)

4. Bochicchio, I, Giorgi, C, Vuk, E: Steady states analysis and exponential stability of an extensible thermoelastic system. Comun. SIMAI Congr. 3, 232-244 (2009)

5. Bochicchio, I, Vuk, E: Buckling and longterm dynamics of a nonlinear model for the extensible beam. Math. Comput. Model. 51, 833-846 (2010)

6. Chen, JS, Ro, WC, Lin, JS: Exact static and dynamic critical loads of a sinusoidal arch under a point force at the midpoint. Int. J. Non-Linear Mech. 44, 66-70 (2009)

7. Coti Zelati, M, Giorgi, C, Pata, V: Steady states of the hinged extensible beam with external load. Math. Models Methods Appl. Sci. 20, 43-58 (2010)

8. Reiss, EL, Matkowsky, BJ: Nonlinear dynamic buckling of a compressed elastic column. Q. Appl. Math. 29, 245-260 (1971)

9. Ball, JM: Initial-boundary value problems for an extensible beam. J. Math. Anal. Appl. 42,61-90 (1973)

10. Woinowsky-Krieger, S: The effect of an axial force on the vibration of hinged bars. J. Appl. Mech. 17, 35-36 (1950)

11. Lazer, AC, McKenna, PJ: Large-amplitude periodic oscillations in suspension bridges: some new connections with nonlinear analysis. SIAM Rev. 32, 537-578 (1990)

12. McKenna, PJ, Walter, W: Nonlinear oscillations in a suspension bridge. Arch. Ration. Mech. Anal. 98, 167-177 (1987)

13. Abdel-Ghaffar, AM, Rubin, LI: Non linear free vibrations of suspension bridges: theory. J. Eng. Mech. 109, 313-329 (1983)

14. Ahmed, NU, Harbi, H: Mathematical analysis of dynamic models of suspension bridges. SIAM J. Appl. Math. 58, 853-874 (1998)

15. Bochicchio, I, Giorgi, C, Vuk, E: Longterm damped dynamics of the extensible suspension bridge. Int. J. Differ. Equ. 2010, Article ID 383420 (2010)

16. Bochicchio, I, Vuk, E: Longtime behavior for oscillations of an extensible viscoelastic beam with elastic external supply. Int. J. Pure Appl. Math. 58, 61-67 (2010)

17. Giorgi, C, Naso, MG: Modeling and steady states analysis of the extensible thermoelastic beam. Math. Comput. Model. 53, 896-908 (2011)

18. Giorgi, C, Pata, V, Vuk, E: On the extensible viscoelastic beam. Nonlinearity 21, 713-733 (2008)

19. An, Y, Fan, X: On the coupled systems of second and fourth order elliptic equations. Appl. Math. Comput. 140 341-351 (2003)

20. Bochicchio, I, Giorgi, C, Vuk, E: Long-term dynamics of the coupled suspension bridge system. Math. Models Methods Appl. Sci. 22, 1250021 (2012)

21. Dickey, RW: Dynamic stability of equilibrium states of the extensible beam. Proc. Am. Math. Soc. 41, 94-102 (1973)

doi:10.1186/1687-2770-2013-204

Cite this article as: Giorgi and Vuk: Steady-state solutions for a suspension bridge with intermediate supports.

Boundary Value Problems 2013 2013:204.

\section{Submit your manuscript to a SpringerOpen ${ }^{\circ}$ journal and benefit from:}

- Convenient online submission

Rigorous peer review

- Immediate publication on acceptance

- Open access: articles freely available online

- High visibility within the field

- Retaining the copyright to your article 